\title{
DISPATCHES
}

\section{Invasive Pneumococcal Disease 3 Years after Introduction of 10-Valent Pneumococcal Conjugate Vaccine, the Netherlands}

\author{
Mirjam J. Knol, Gertjan H.J. Wagenvoort, \\ Elisabeth A.M. Sanders, Karin Elberse, \\ Bart J. Vlaminckx, Hester E. de Melker, \\ Arie van der Ende
}

Three years after a 7-valent pneumococcal conjugate vaccine was replaced by a 10 -valent pneumococcal conjugate vaccine in the Netherlands, we observed a decrease in incidence of invasive pneumococcal disease caused by Streptococcus pneumoniae serotypes 1,5 , and 7F. Our data do not support or exclude cross-protection against serotype 19A.

A 7-valent pneumococcal conjugate vaccine (PCV7) was first used in the Netherlands in June 2006 in a 3 +1 schedule for protection against invasive pneumococcal disease (IPD). A switch to 10-valent pneumococcal conjugate vaccine (PCV10) was made in May 2011. There were no catch-up campaigns; children vaccinated with PCV7 completed their series with PCV7. Vaccination coverage has been $94 \%-95 \%$ since PCV7 introduction (1).

After PCV7 introduction, vaccine-type IPD incidence decreased for all age groups (2). However, nasopharyngeal carriage and incidence of IPD caused by nonvaccine serotypes increased $(2,3)$. Nevertheless, a $7 \%$ decrease in overall IPD incidence was observed 4 years after PCV7 introduction (2).

Studies assessing the effect of PCV10 when used in national vaccination programs are limited. Two studies on PCV10 effectiveness in children suggested cross-protection against vaccine-related serotypes, including 19A (4,5). No studies have been published on herd effects of PCV10 in unvaccinated persons. We report the effect of switching from PCV7 to PCV10 on IPD incidence in the Netherlands.

Author affiliations; National Institute for Public Health and the Environment, Bilthoven, the Netherlands (M.J. Knol,

E.A.M. Sanders, K. Elberse, H.E. de Melker); St. Antonius Hospital, Nieuwegein, the Netherlands (G.H.J. Wagenvoort, B.J. Vlaminckx); University Medical Center Utrecht, Utrecht, the Netherlands (E.A.M. Sanders); Amsterdam Medical Center, Amsterdam, the Netherlands (A. van der Ende); Netherlands Reference Laboratory for Bacterial Meningitis, Amsterdam (A. van der Ende)

DOI: http://dx.doi.org/10.3201/eid2111.140780

\section{The Study}

We used data for June 2004-May 2014 from a sentinel laboratory surveillance system, as described (2). These data cover $\approx 25 \%$ of the population of the Netherlands. Cumulative incidence ratios (CIRs) with $95 \%$ CIs were calculated for comparisons of pre-PCV7 (June 2004-May 2006), prePCV10 (June 2009-May 2011), early post-PCV10 (June 2011-May 2013) and 3-year-post PCV10 (June 2013-May 2014) periods. We discerned IPD caused by PCV7 (4, 6B, $9 \mathrm{~V}, 14,18 \mathrm{C}, 19 \mathrm{~F}, 23 \mathrm{~F}$ ), non-PCV7, PCV10-7 (present in PCV10 but not PCV7; 1, 5, 7F), non-PCV10, and PCV10 -related $(6 \mathrm{~A}, 6 \mathrm{C}, 6 \mathrm{D}, 7 \mathrm{~A}, 7 \mathrm{~B}, 7 \mathrm{C}, 9 \mathrm{~A}, 9 \mathrm{~L}, 9 \mathrm{~N}, 18 \mathrm{~A}, 18 \mathrm{~B}$, 18F, 19A, 19B, 19C, 23A, 23B) serotypes.

We used nationwide laboratory surveillance data for children $<5$ years of age available from 2006 to compare IPD incidence rates for a PCV7-eligible cohort (children born March 2008-Feb 2011, $\geq 3$ months of age, and given a diagnosis of IPD before June 2011) and a PCV10-eligible cohort (children born March 2011-Feb 2014, $>3$ months of age, and given a diagnosis of IPD before June 2014). Incidence rate ratios (IRRs) with $95 \%$ CIs and $p$ values were calculated. Differences between IRRs were tested by calculating $p$ values for interaction between birth cohort and serotype; the IRR for serotypes not related to PCV10 was used as reference.

A total of 6,292 IPD cases were included in sentinel surveillance during June 2004-May 2014. By 2009-2011, overall IPD incidence had decreased by $57 \%$ for children $<2$ years of age, $47 \%$ for children $2-4$ years of age, and $22 \%$ for persons $\geq 65$ years of age (Figure 1; Table 1). No further decrease was observed during 2011-2014 for persons $\geq 65$ years of age. PCV7 IPD incidence decreased for all age groups, and the decrease continued and showed an overall reduction of $90 \%$ by 2013-2014 (Figure 2, panel A). Non-PCV7 IPD incidence increased by $38 \%$ for all age groups from 2004-2006 to 2009-2011 (Figure 2, panel B; Table 1).

Overall PCV10-7 IPD incidence increased slightly after PCV7 introduction (CIR 1.21, 95\% CI 1.02-1.43) (Figure 2 panel C; Table 1). For children $<2$ years of age, PCV10-7 IPD incidence decreased 2 years after the switch to PCV10, although not significantly, because of a low number of cases (CIR $0.51,95 \%$ CI $0.13-2.02$ ). In the third year (2013-2014) after PCV10 introduction, no IPD cases were caused by serotypes 1,5 , and $7 \mathrm{~F}$ in children $<2$ years of age. For other age groups, PCV10-7 IPD incidence did 


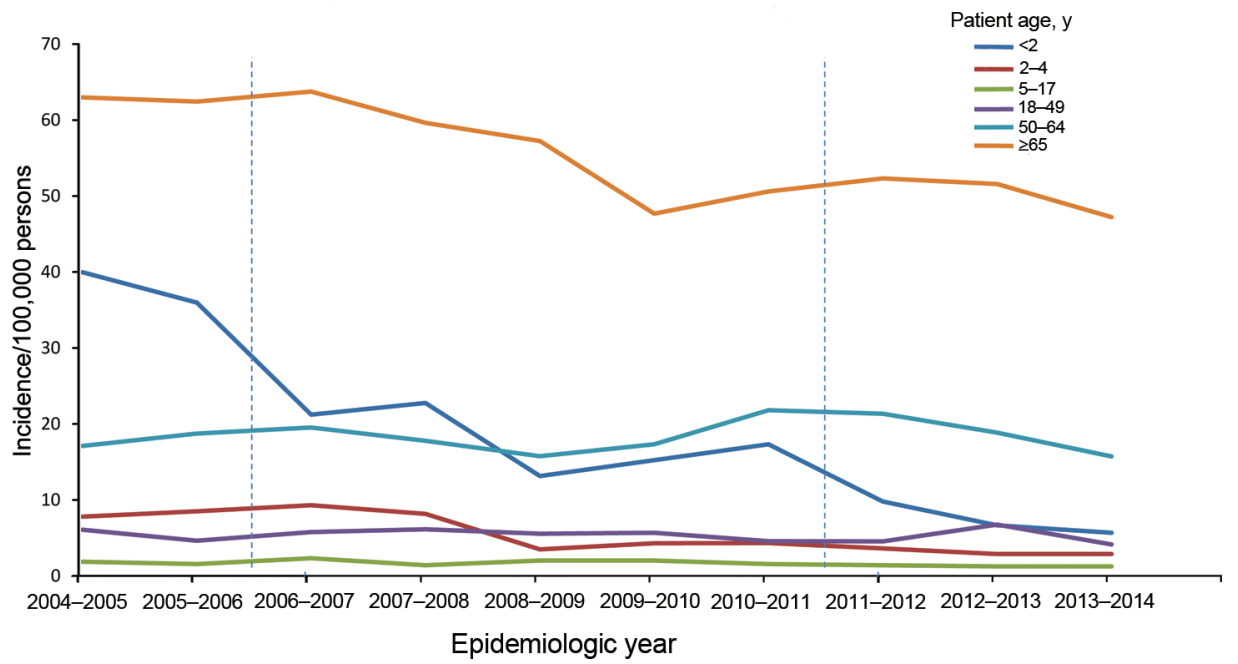

Figure 1. Age-specific incidence of invasive pneumococcal disease caused by any serotype of Streptococcus pneumoniae per epidemiologic year (June-May), the Netherlands. Vertical dashed lines indicate introduction of 7-valent pneumococcal conjugate vaccine in June 2006 and 10-valent pneumococcal conjugate vaccine in May 2011. Incidences are based on sentinel surveillance data and extrapolated to the national level.

not change in the 2 years after PCV10 introduction, but in the third year, incidence decreased by $42 \%$ and $47 \%$ for persons 18-49 years of age and 50-64 years of age, respectively; a $25 \%$ decrease was observed for persons $\geq 65$ years of age (Figure 2 panel C; Table 1). Non-PCV10 IPD incidence increased for most age groups in the 2 years after PCV10 introduction (overall CIR 1.25, 95\% CI 1.13-1.38) but did not increase further in 2013-2014 (Figure 2, panel D; Table 1), partly because of a decrease in 19A IPD (Figure 2, panel E; Table 1).

The IPD incidence rate for the PCV10-eligible cohort was lower than that for the PCV7-eligible cohort for PCV10 7 serotypes (IRR 0.04, 95\% CI 0.01-0.27), PCV10-related serotype 19A (IRR $0.38,95 \%$ CI 0.19-0.77), and serotypes not related to PCV10 (IRR 0.67, 95\% CI 0.46-0.99)
(Table 2). The decrease in PCV10-7 IPD was greater than that for PCV10-unrelated serotypes $\left(\mathrm{p}_{\text {interaction }}=0.005\right)$. However, IRRs for PCV10-related IPD and specifically serotype 19A were not different from the IRR for PCV10unrelated IPD $\left(\mathrm{p}_{\text {interaction }}=0.229 / 0.165\right)$.

\section{Conclusions}

We observed a decrease in PCV7-type IPD $\geq 8$ years after PCV7 introduction for all age groups. However, this decrease was lessened by an increase in non-vaccine-type IPD, a finding similar to that reported in other countries $(6,7)$. There was an overall $80 \%$ decrease in IPD incidence for children $<5$ years of age and a $25 \%$ decrease for persons $\geq 65$ years of age.

PCV10introduction caused a decrease in PCV10-7IPD incidence in PCV10-eligible children, providing evidence
A

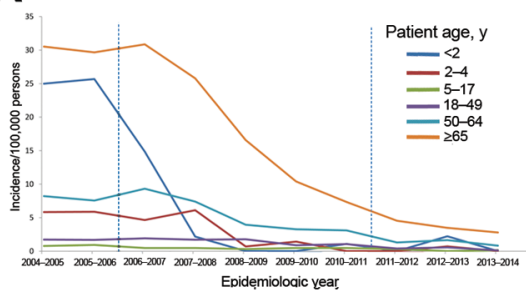

D

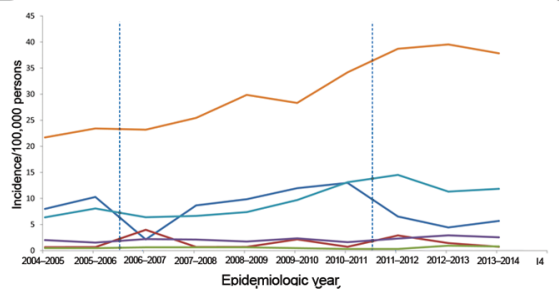

B

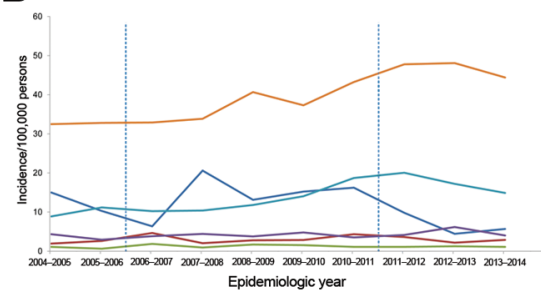

E

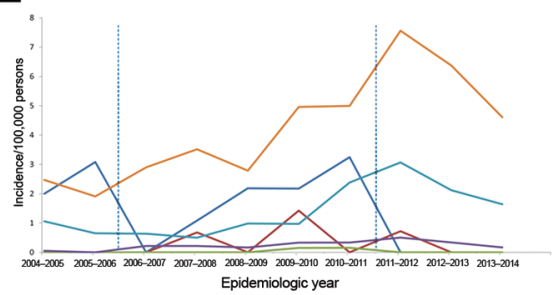

C

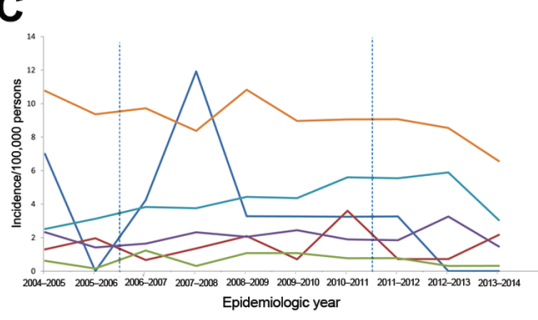

Epidemiologic year

Figure 2. Age-specific incidence of invasive pneumococcal disease caused by different Streptococcus pneumoniae serotypes per epidemiologic year (June-May), the Netherlands. A) 7-valent pneumococcal conjugate vaccine (PCV7) serotypes; B) non-PCV7 serotypes; C) PCV10-7 serotypes; D) non-PCV10 serotypes; and E) serotype 19A. Vertical dashed lines indicate introduction of PCV7 in June 2006 and PCV10 in May 2011. Incidences are based on sentinel surveillance data and extrapolated to the national level. 
for a direct effect from PCV10. Potential cross-protection of PCV10 against serotype 19A, as corroborated by a case-control study showing $82 \%$ effectiveness against 19A IPD (5), is still debated. In our study, the incidence rate for 19A IPD was lower in the PCV10-eligible cohort than the PCV7-eligible cohort, but the decrease in 19A IPD was not different from the decrease in PCV10unrelated IPD, which precludes drawing conclusions about cross-protection against 19A IPD. In addition, 19A carriage had already decreased in toddlers before PCV10 introduction $(8)$.

We observed a decrease in non-PCV10 IPD in the PCV10-eligible cohort but have no indication that surveillance sensitivity changed over time. The decrease might be caused by natural fluctuations or different viral seasons (9). A study in Canada reported lower incidence

\begin{tabular}{|c|c|c|c|c|c|c|c|}
\hline \multirow[b]{2}{*}{$\begin{array}{l}\text { Serotype and } \\
\text { patient age } \\
\text { group, y }\end{array}$} & \multicolumn{4}{|c|}{ No. cases } & \multicolumn{3}{|c|}{ Comparison, CIR $(95 \% \mathrm{Cl})$} \\
\hline & $\begin{array}{l}\text { Pre-PCV7, } \\
2004-2006\end{array}$ & $\begin{array}{l}\text { Pre-PCV10, } \\
2009-2011\end{array}$ & $\begin{array}{c}\text { Early post- } \\
\text { PCV10, } \\
\text { 2011-2013 }\end{array}$ & $\begin{array}{c}3 \text { years post- } \\
\text { PCV10, } \\
2013-2014\end{array}$ & $\begin{array}{l}\text { Pre-PCV10 vs. } \\
\text { pre-PCV7 }\end{array}$ & $\begin{array}{c}\text { Early post-PCV10 } \\
\text { vs. pre-PCV10 }\end{array}$ & $\begin{array}{l}3 \text { years post- } \\
\text { PCV10 vs. early } \\
\text { post-PCV10 }\end{array}$ \\
\hline \multicolumn{8}{|l|}{ All } \\
\hline$<2$ & 75 & 30 & 15 & 5 & $0.43(0.28-0.65)$ & $0.51(0.27-0.94)$ & $0.69(0.25-1.90)$ \\
\hline $2-4$ & 25 & 12 & 9 & 4 & $0.53(0.27-1.05)$ & $0.75(0.32-1.79)$ & $0.89(0.27-2.89)$ \\
\hline $5-17$ & 22 & 23 & 17 & 8 & $1.04(0.58-1.87)$ & $0.75(0.40-1.40)$ & $0.95(0.41-2.20)$ \\
\hline $18-49$ & 197 & 184 & 201 & 73 & $0.96(0.78-1.17)$ & $1.10(0.90-1.34)$ & $0.73(0.56-0.96)$ \\
\hline $50-64$ & 273 & 326 & 341 & 134 & $1.09(0.93-1.28)$ & $1.03(0.88-1.19)$ & $0.78(0.64-0.95)$ \\
\hline$\geq 65$ & 717 & 622 & 703 & 338 & $0.78(0.70-0.87)$ & $1.06(0.95-1.18)$ & $0.91(0.80-1.03)$ \\
\hline $\bar{T}$ otal & 1,309 & 1,197 & 1,286 & 562 & $0.90(0.83-0.97)$ & $1.06(0.98-1.15)$ & $0.87(0.79-0.96)$ \\
\hline \multicolumn{8}{|l|}{ PCV7 } \\
\hline$<2$ & 50 & 1 & 2 & 0 & $0.02(0.00-0.16)$ & $2.03(0.18-22.33)$ & NC \\
\hline $2-4$ & 18 & 2 & 1 & 0 & $0.12(0.03-0.53)$ & $0.50(0.05-5.55)$ & NC \\
\hline $5-17$ & 11 & 6 & 2 & 1 & $0.54(0.20-1.47)$ & $0.34(0.07-1.67)$ & $1.01(0.09-11.1)$ \\
\hline $18-49$ & 63 & 35 & 17 & 2 & $0.57(0.38-0.86)$ & $0.49(0.27-0.87)$ & $0.24(0.05-1.03)$ \\
\hline $50-64$ & 120 & 53 & 25 & 7 & $0.40(0.29-0.56)$ & $0.46(0.29-0.74)$ & $0.56(0.24-1.29)$ \\
\hline$\geq 65$ & 344 & 112 & 54 & 20 & $0.29(0.24-0.36)$ & $0.45(0.33-0.62)$ & $0.70(0.42-1.17)$ \\
\hline Total & 606 & 209 & 101 & 30 & $0.34(0.29-0.40)$ & $0.48(0.38-0.61)$ & $0.59(0.39-0.89)$ \\
\hline \multicolumn{8}{|l|}{ Non-PCV7 } \\
\hline$<2$ & 25 & 29 & 13 & 5 & $1.24(0.73-2.12)$ & $0.45(0.24-0.87)$ & $0.80(0.28-2.23)$ \\
\hline $2-4$ & 7 & 10 & 8 & 4 & $1.57(0.60-4.13)$ & $0.80(0.32-2.04)$ & $1.00(0.30-3.32)$ \\
\hline $5-17$ & 11 & 17 & 15 & 7 & $1.54(0.72-3.30)$ & $0.89(0.44-1.78)$ & $0.94(0.38-2.31)$ \\
\hline $18-49$ & 134 & 149 & 184 & 71 & $1.14(0.90-1.44)$ & $1.24(1.00-1.54)$ & $0.78(0.59-1.02)$ \\
\hline $50-64$ & 153 & 273 & 316 & 127 & $1.63(1.34-1.99)$ & $1.14(0.97-1.33)$ & $0.80(0.65-0.98)$ \\
\hline$\geq 65$ & 373 & 510 & 649 & 318 & $1.24(1.08-1.41)$ & $1.19(1.06-1.34)$ & $0.93(0.81-1.06)$ \\
\hline Total & 703 & 988 & 1,185 & 532 & $1.38(1.26-1.52)$ & $1.19(1.09-1.29)$ & $0.89(0.81-0.99)$ \\
\hline \multicolumn{8}{|l|}{ PCV10-7 } \\
\hline$<2$ & 7 & 6 & 3 & 0 & $0.92(0.31-2.73)$ & $0.51(0.13-2.02)$ & $\mathrm{NC}$ \\
\hline $2-4$ & 5 & 6 & 2 & 3 & $1.32(0.40-4.33)$ & $0.34(0.07-1.66)$ & $3.00(0.50-18.0)$ \\
\hline $5-17$ & 5 & 12 & 7 & 2 & $2.40(0.84-6.81)$ & $0.59(0.23-1.49)$ & $0.58(0.12-2.77)$ \\
\hline $18-49$ & 69 & 78 & 91 & 26 & $1.16(0.84-1.60)$ & $1.18(0.87-1.59)$ & $0.58(0.37-0.89)$ \\
\hline $50-64$ & 43 & 83 & 97 & 26 & $1.77(1.22-2.55)$ & $1.15(0.86-1.54)$ & $0.53(0.35-0.82)$ \\
\hline$\geq 65$ & 115 & 114 & 119 & 47 & $0.90(0.69-1.16)$ & $0.98(0.76-1.26)$ & $0.75(0.53-1.05)$ \\
\hline $\bar{T}$ otal & 244 & 299 & 319 & 104 & $1.21(1.02-1.43)$ & $1.06(0.90-1.24)$ & $0.65(0.52-0.81)$ \\
\hline \multicolumn{8}{|l|}{ Non-PCV10 } \\
\hline$<2$ & 18 & 23 & 10 & 5 & NA & $0.44(0.21-0.92)$ & $1.03(0.35-3.03)$ \\
\hline $2-4$ & 2 & 4 & 6 & 1 & NA & $1.51(0.43-5.35)$ & $0.33(0.04-2.77)$ \\
\hline $5-17$ & 6 & 5 & 8 & 5 & NA & $1.61(0.53-4.93)$ & $1.26(0.41-3.85)$ \\
\hline $18-49$ & 65 & 71 & 93 & 45 & NA & $1.32(0.97-1.80)$ & $0.98(0.68-1.39)$ \\
\hline $50-64$ & 110 & 190 & 219 & 101 & NA & $1.13(0.93-1.37)$ & $0.92(0.72-1.16)$ \\
\hline$>65$ & 258 & 396 & 530 & 271 & NA & $1.25(1.10-1.43)$ & $0.97(0.84-1.12)$ \\
\hline Total & 459 & 689 & 866 & 428 & NA & $1.25(1.13-1.38)$ & $0.98(0.88-1.11)$ \\
\hline \multicolumn{8}{|l|}{$19 A$} \\
\hline$<2$ & 5 & 5 & 0 & 0 & $1.07(0.31-3.70)$ & $0.00(\mathrm{NC})$ & NC \\
\hline $2-4$ & 0 & 2 & 1 & 0 & NC & $0.50(0.05-5.55)$ & NC \\
\hline $5-17$ & 0 & 2 & 0 & 0 & NC & $0.00(\mathrm{NC})$ & $\mathrm{NC}$ \\
\hline $18-49$ & 1 & 12 & 15 & 3 & $12.3(1.60-94.6)$ & $1.26(0.59-2.69)$ & $0.40(0.12-1.39)$ \\
\hline $50-64$ & 13 & 28 & 44 & 14 & $1.97(1.02-3.81)$ & $1.54(0.96-2.48)$ & $0.63(0.35-1.15)$ \\
\hline$\geq 65$ & 25 & 63 & 94 & 33 & $2.28(1.43-3.62)$ & $1.40(1.02-1.92)$ & $0.66(0.45-0.99)$ \\
\hline$\overline{\text { Total }}$ & 44 & 112 & 154 & 50 & $2.50(1.77-3.55)$ & $1.36(1.07-1.74)$ & $0.65(0.47-0.89)$ \\
\hline
\end{tabular}

*PCV7, 7-valent pneumococcal conjugate vaccine; PCV10-7, serotypes present in PCV10 but not PCV7 (3 additional serotypes); PCV10, 10-valent pneumococcal conjugate vaccine; CIR, cumulative incidence ratio; NC, not calculated (numbers too low to be informative or relevant). NA, not applicable. 
rates for 19A IPD and other non-vaccine-type IPD in a PCV10-eligible cohort (4). It was hypothesized that lower antibody levels induced by PCV10 $(10,11)$ might lead to smaller disturbances of the nasopharyngeal niche and replacement by new serotypes against which there is no immunity, which might result in a lower incidence of non-PCV10 IPD. However, a randomized controlled trial showed similar carriage rates for non-PCV10 serotypes, including 19A, for infants vaccinated with PCV7 and those vaccinated with PCV10 (12).

In the third year after PCV10 introduction, PCV10-7 IPD incidence also decreased in nonvaccinated age groups, which might indicate herd effects. After PCV7 introduction, herd effects appeared after 3 years (13). Non-PCV10 IPD incidence did not increase in the second and third years after PCV10 introduction, which was partially caused by a reduction in 19A IPD. Longer follow-up times are needed to distinguish whether these observations were caused by cross-protection against 19A in children through herd effects of PCV10, reduced nonvaccine serotype replacement by PCV10, or temporal fluctuations.

We used data from a stable surveillance system with constant coverage over time; age and serotype data were nearly complete $(99.9 \%)$. However, a limitation of our

\begin{tabular}{|c|c|c|c|c|c|}
\hline Variable & PCV7-eligible cohort & PCV10-eligible cohort & IRR $(95 \% \mathrm{Cl}) \dagger$ & $\begin{array}{l}\text { Exact } p \\
\text { value }\end{array}$ & $\begin{array}{l}p \text { value for } \\
\text { interaction } \ddagger\end{array}$ \\
\hline Birth cohort & 2008 Mar 1-2011 Feb 28 & 2011 Mar 1-2014 Feb 28 & NA & NA & NA \\
\hline Observation period & 2008 Jun 1-2011 May 31 & 2011 Jun 1-2014 May 31 & NA & NA & NA \\
\hline Persons at risk & 550,297 & 537,071 & NA & NA & NA \\
\hline Person-years at risk & 822,100 & 814,980 & NA & NA & NA \\
\hline \multicolumn{6}{|c|}{$\begin{array}{l}\text { No. (\%) IPD cases per } 100,000 \text { persons } \\
\text { Serotypes }\end{array}$} \\
\hline PCV7§ & $5(0.6)$ & $0(0.0)$ & $\mathrm{NC}$ & 0.063 & NC \\
\hline 6B & 2 & 0 & $\mathrm{NC}$ & NC & NC \\
\hline $18 \mathrm{C}$ & 2 & 0 & $\mathrm{NC}$ & $\mathrm{NC}$ & NC \\
\hline $19 F$ & 1 & 0 & NC & NC & NC \\
\hline PCV10-7 & $27(3.3)$ & $1(0.1)$ & $0.04(0.01-0.27)$ & $<0.001$ & 0.005 \\
\hline 1 & 2 & 0 & NC & NC & NC \\
\hline 5 & 2 & 0 & NC & NC & NC \\
\hline $7 \mathrm{~F}$ & 23 & 1 & $0.04(0.01-0.32)$ & $<0.001$ & 0.009 \\
\hline PCV10-related§ & $33(4.0)$ & $14(1.7)$ & $0.43(0.23-0.80)$ & 0.006 & 0.229 \\
\hline $6 \mathrm{~A}$ & 2 & 0 & NC & NC & NC \\
\hline $6 \mathrm{C}$ & 0 & 1 & $\mathrm{NC}$ & NC & NC \\
\hline $9 \mathrm{~N}$ & 0 & 1 & NC & NC & NC \\
\hline $19 A$ & 29 & 11 & $0.38(0.19-0.77)$ & 0.005 & 0.165 \\
\hline $23 \mathrm{~A}$ & 1 & 1 & NC & NC & NC \\
\hline 23B & 1 & 0 & NC & NC & NC \\
\hline PCV10-unrelated & $63(7.7)$ & $42(5.2)$ & $0.67(0.46-0.99)$ & 0.045 & Reference \\
\hline 3 & 4 & 0 & NC & NC & NC \\
\hline 8 & 3 & 2 & NC & NC & NC \\
\hline $10 \mathrm{~A}$ & 14 & 16 & $\mathrm{NC}$ & $\mathrm{NC}$ & $\mathrm{NC}$ \\
\hline $11 \mathrm{~A}$ & 3 & 0 & NC & NC & $\mathrm{NC}$ \\
\hline $12 \mathrm{~F}$ & 2 & 2 & NC & NC & NC \\
\hline $15 \mathrm{~A}$ & 1 & 0 & NC & $\mathrm{NC}$ & NC \\
\hline 15B & 3 & 1 & NC & $\mathrm{NC}$ & $\mathrm{NC}$ \\
\hline $15 \mathrm{C}$ & 1 & 4 & NC & $\mathrm{NC}$ & $\mathrm{NC}$ \\
\hline $16 \mathrm{~F}$ & 4 & 1 & NC & NC & NC \\
\hline $17 \mathrm{~F}$ & 3 & 1 & NC & $\mathrm{NC}$ & $\mathrm{NC}$ \\
\hline $22 \mathrm{~F}$ & 5 & 3 & NC & $\mathrm{NC}$ & $\mathrm{NC}$ \\
\hline 24B & 0 & 1 & NC & $\mathrm{NC}$ & $\mathrm{NC}$ \\
\hline $24 \mathrm{~F}$ & 3 & 1 & NC & $\mathrm{NC}$ & $\mathrm{NC}$ \\
\hline 27 & 2 & 1 & NC & NC & NC \\
\hline $33 \mathrm{~F}$ & 11 & 6 & NC & NC & NC \\
\hline 34 & 1 & 0 & NC & $\mathrm{NC}$ & $\mathrm{NC}$ \\
\hline $35 B$ & 0 & 1 & NC & NC & NC \\
\hline $35 \mathrm{~F}$ & 2 & 1 & NC & NC & NC \\
\hline 38 & 1 & 1 & NC & NC & $\mathrm{NC}$ \\
\hline Total & $128(15.6)$ & $57(7.0)$ & $0.45(0.33-0.61)$ & $<0.001$ & NA \\
\hline
\end{tabular}


study was the ecologic design. Thus, one should be cautious in interpreting findings as causally related to vaccination. Also, we have limited data on IPD before PCV7 introduction and after the switch to PCV10.

In conclusion, PCV10 introduction in 2011 decreased vaccine-type IPD incidence in targeted birth cohorts. Three years after introduction, herd effects became apparent. Stabilization of non-PCV10 IPD in the second and third years after PCV10 introduction might indicate reduced serotype replacement by PCV10 or cross-protection against 19A. However, we cannot make firm conclusions on crossprotection of PCV10 against serotype 19A. Continued surveillance of serotype-specific IPD is crucial for evaluating long-term effects of pneumococcal conjugate vaccines in human populations.

Dr. Knol is a senior epidemiologist at the Center for Infectious Disease Control of the National Institute of Public Health, Bilthoven, the Netherlands. Her research interests are epidemiology and surveillance of vaccine-preventable diseases, in particular IPD.

\section{References}

1. van Lier EA, Oomen PJ, Mulder M, Conyn-van Spaendonck MA, Drijfhout IH, de Hoogh PA, et al. Vaccination coverage, National Immunization Program, the Netherlands; 2013 [in Dutch] [cited 2015 Jul 15]. http://www.rivm.nl/en/Documents_and_publications/ Scientific/Reports/2013/juni/Immunisation_coverage_National_Immunisation_Programme_in_the_Netherlands_Year_of_report_2013

2. van Deursen AM, van Mens SP, Sanders EA, Vlaminckx BJ, de Melker HE, Schouls LM, et al. Invasive pneumococcal disease and 7-valent pneumococcal conjugate vaccine, the Netherlands. Emerg Infect Dis. 2012;18:1729-37. http://dx.doi.org/10.3201/ eid1811.120329

3. Spijkerman J, Prevaes SM, van Gils EJ, Veenhoven RH, Bruin JP, Bogaert D, et al. Long-term effects of pneumococcal conjugate vaccine on nasopharyngeal carriage of $S$. pneumoniae, $S$. aureus, H. influenzae and M. catarrhalis. PLoS ONE. 2012;7:e39730. http://dx.doi.org/10.1371/journal.pone.0039730

4. De Wals P, Lefebvre B, Defay F, Deceuninck G, Boulianne N. Invasive pneumococcal diseases in birth cohorts vaccinated with PCV-7 and/or PHiD-CV in the province of Quebec, Canada. Vaccine. 2012;30:6416-20. http://dx.doi.org/10.1016/ j.vaccine.2012.08.017

5. Domingues CM, Verani JR, Montenegro Renoiner EI, de Cunto Brandileone MC, Flannery B, de Oliveira LH, et al. Effectiveness of ten-valent pneumococcal conjugate vaccine against invasive pneumococcal disease in Brazil: a matched case-control study. Lancet Respir Med. 2014;2:464-71.
http://dx.doi.org/10.1016/S2213-2600(14)70060-8

6. Feikin DR, Kagucia EW, Loo JD, Link-Gelles R, Puhan MA, Cherian T, et al. Serotype-specific changes in invasive pneumococcal disease after pneumococcal conjugate vaccine introduction: a pooled analysis of multiple surveillance sites. PLoS Med. 2013;10:e1001517. http://dx.doi.org/10.1371/ journal.pmed.1001517

7. Myint TT, Madhava H, Balmer P, Christopoulou D, Attal S, Menegas D, et al. The impact of 7-valent pneumococcal conjugate vaccine on invasive pneumococcal disease: a literature review. Adv Ther. 2013;30:127-51. http://dx.doi.org/10.1007/ s12325-013-0007-6

8. Bosch AA, Veenhoven R, Bruin JP, Wijmenga-Monsuur AJ, Trzcinski K, Wyllie A, et al. A new balance in carriage of non-vaccine pneumococcal serotypes 6.5 years after PCV7 implementation. In: Abstracts of the 9th International Symposium on Pneumococci and Pneumococcal Diseases, Hyberadad, India, March 9-13, 2014. Geneva: Kenes International; 2014.

Abstract no. ISPDD no. 0278.

9. Jansen AG, Sanders EA, Hoes AW, van Loon AM, Hak E. Influenza- and respiratory syncytial virus-associated mortality and hospitalisations. Eur Respir J. 2007;30:1158-66. http://dx.doi.org/ 10.1183/09031936.00034407

10. van den Bergh MR, Spijkerman J, Francois N, Swinnen K, Borys D, Schuerman L, et al. Immunogenicity, safety, and reactogenicity of the 10 -valent pneumococcal nontypeable Haemophilus influenzae protein D conjugate vaccine and DTPa-IPV-Hib when coadministered as a 3-dose primary vaccination schedule in The Netherlands: a randomized controlled trial. Pediatr Infect Dis J. 2011;30:e170-8. http://dx.doi.org/ 10.1097/INF.0b013e31821a0614

11. Vesikari T, Wysocki J, Chevallier B, Karvonen A, Czajka H, Arsene JP, et al. Immunogenicity of the 10-valent pneumococcal non-typeable Haemophilus influenzae protein D conjugate vaccine (PHiD-CV) compared to the licensed 7vCRM vaccine. Pediatr Infect Dis J. 2009;28(Suppl):S66-76. http://dx.doi.org/10.1097/ INF.0b013e318199f8ef

12. van den Bergh MR, Spijkerman J, Swinnen KM, Francois NA, Pascal TG, Borys D, et al. Effects of the 10-valent pneumococcal nontypeable Haemophilus influenzae protein D-conjugate vaccine on nasopharyngeal bacterial colonization in young children: a randomized controlled trial. Clin Infect Dis. 2013;56:e30-9. http://dx.doi.org/10.1093/cid/cis922

13. Rodenburg GD, de Greeff SC, Jansen AG, de Melker HE, Schouls LM, Hak E, et al. Effects of pneumococcal conjugate vaccine 2 years after its introduction, the Netherlands. Emerg Infect Dis. 2010;16:816-23. http://dx.doi.org/10.3201/ eid1605.091223

Address for correspondence: Mirjam J. Knol, National Institute for Public Health and the Environment, PO Box 1, 3720 BA Bilthoven, the Netherlands; email: mirjam.knol@rivm.nl

\section{Like our podcasts?}

Sign up to receive emaill announcements when a new podcast is available. www.cdc.gov/eid/subscribe.htm

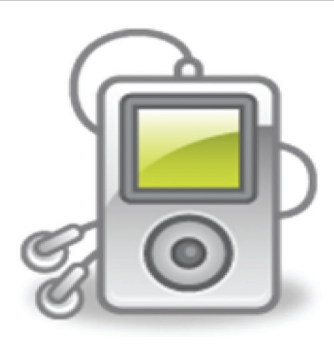

